\title{
Segmentation and Visualization of Human Coronary Artery Trees from CTA Datasets
}

\author{
Qian Huang \\ Computer Science Department \\ Wright State University, United States
}

\begin{abstract}
The volume information extracted from computed tomography angiogram is very useful for cardiologists to diagnose various diseases. An approach is presented to segment human coronary artery trees from the volumetric datasets. The coronary arteries' surfaces are recovered by triangle mesh with the boundary points extracted from the coronary artery voxels segmented. The positions where the calcified plaques occur are identified by mapping the intensities of boundary points of the coronary artery trees on the triangle meshed surfaces. If different values of the computed maximum principle curvatures of boundary points surrounding the lumen cross section are mapped on the triangle meshed surfaces of the segmented coronary artery trees, the cross section structure of the coronary artery lumen segment is noncircular cross section structure.
\end{abstract}

Keywords: Segmentation, visualization, coronary arteries, calcified plaque, maximum principle curvature.

\section{INTRODUCTION}

Computed tomography angiogram (CTA) [1] plays a significant role as a clinical tool in the diagnosis of the coronary artery diseases. It provides three-dimensional information [2], allows for a better understanding of cardiac three-dimensional anatomy [4], [5], medical diagnosis [6], and ongoing investigations of acute and chronic coronary heart diseases [7]. The noninvasive [9] manner empowered with the visualization of vessels attracts cardiologists to apply it in clinical environments. Segmenting and visualizing coronary artery trees and deriving quantitative data [18] from CTA image datasets for inspecting the coronary lesions is what many algorithms' goal to focus on.

Several algorithms have been developed to segment and visualize vessels in three dimensions [3], for instance, level set method [10], active contour algorithm [14], vesselness measurement [11], [12], expectation maximization estimation algorithm [15], moment-based shape analysis for voxel clusters [16], shape model based algorithms such as tubular model in three dimensions [17], the algorithm of combining graph-cuts and robust kernel regression to segment coronary lumens [18], [19]. The standard marching cube algorithm [21] generates a high resolution isosurface with an isovalue of image intensity to represent the object's surface. The vertices of the set of triangles build the boundary point cloud of the object. A gradient based algorithm is used to extract vessel boundaries, the vessel boundaries are represented as an unstructured point cloud in three-dimensional image space. The locations of the vessel boundaries are defined among a set of voxels with the maximum gradient magnitude along the gradient direction. In this work, this algorithm will be applied to produce a boundary point cloud representing the coronary artery boundaries of a human heart.

The existences of the calcified and soft plaques are harmful to the health of a human body [22-24]. Calcified plaques are with high intensities [15]. Assuming voxels' intensities of the segmented coronary arteries are a Gaussian distribution brings out calcified plaques' being identified. The computed mean value plus three standard deviations of the intensities is the threshold value to recognize the calcified plaques [25]. Stenoses or soft plaques are studied to be detected with profiles of artery lumen sectional area or vessel radiuses along the vessels' centerlines [15], [27], [30]. The detections of the artery lumen, calcified and soft plaques or stenoses are clinical useful [31-33]. A coronary artery segmentation method is presented in this work to segment the voxels representing the coronary artery trees from CTA datasets. A triangle mesh method is used to recover the coronary artery surface from those detected vessels' boundary points. The positions of calcified plaques can be identified from the recovered triangle surfaces. The computed maximum principle curvatures of the coronary boundary points are mapped on the triangle surfaces.

\section{SEGMENT THE CORONARY ARTERIES WITH MULTIPLE ISOVALUES}

\subsection{Downsample the CTA Datasets}

Similarly as [8], the sampling theorem in three dimensions is applied in this work. The discrete image dataset is obtained by sampling a continuous function $f_{c}(x, y, z)$ on a lattice with interval $(X, Y, Z)$. For different sampling rate of a continuous function, the lattice interval $(X, Y, Z)$ takes different values. Different lattice interval value represents different voxel size. Then for the same continuous function, the discrete image dataset, when sampled at lower sampling rate, has larger lattice interval, therefore larger voxel size. For the same spatial dimensions, if the discrete image dataset is sampled at high sampling rate, the voxel size is smaller, and the number of the resulted voxels is greater than those sampled at low sampling rate

According to marching cube algorithm, to compute the vessel boundary points from the original size of the CTA dataset, each voxel has to be computed. The extracted boundary point cloud includes the boundary points of arteries, chambers and other tissue structures. In a discrete image dataset, the vessels and other tissue structures are expressed as connected voxels. For the original image dataset, the number of voxels representing the vessels and other tissue structures is greater than those of image dataset with low sampling rate. Then the resulted boundary points from the original size of CTA dataset are more than those from the downsampled CTA dataset.

Consequently, the amount of memory space involved for computing a downsampled CTA dataset is decreased. To downsample the image dataset, the image dataset is convolved with a sinc function and the original continuous function is 
reconstructed, so that it can be resampled. The sinc function has infinite support, therefore, the windowed sinc function has to be used, which truncates the sinc function by multiplying it with a window function. After downsampling the volumetric image dataset, the size of the dataset is changed to $1 / 3$ of the original image size in both rows and columns, respectively, and 1/1.6 of the original image dataset size in the number of slices.

\subsection{Detect Coronary Artery Regions}

Computation of all voxels in each image slice will produce many boundary points including other tissue structures besides the boundaries of the vessels, and the involved memory is of large amount. It is better to segment the regions containing coronary arteries for the extraction of their boundary points. Mathematical morphological operators can cluster a set of connected pixels with a structure element. In this work, such operators are applied to detect the coronary artery regions.

Dilation morphological operation is to move a structure element inside the region of interest, and outputs the locus of the pixels covered by the structure element when its center moves inside the region of interest. The intensities of the artery regions are higher compared with those of the heart muscles. For each image slice, its average intensity is computed. If the pixels' intensities in each image slice are higher than the average intensity plus or minus a small offset defined by the user for segmenting different coronary artery trees, the center of a $3 \times 3$ square structure element is placed on them. All the pixels covered by this square are defined as the foreground region. Erode this foreground region with an $8 \times 8$ square when the downsampled dataset is being processed and with a $24 \times 24$ square when an original dataset is being operated. Small regions of foreground voxels can be removed via this operation. The resulted erosion set is dilated with a $4 \times 4$ square (with a downsampled dataset) or $12 \times 12$ square (with an original dataset) again. The difference of the foreground region and the resulted set is the region where coronary arteries exist. With this region, the produced boundaries belong to other tissue structures and the involved memory can be reduced. An image slice as an instance of the coronary artery region detected is shown in Figure. 1 (a).

\subsection{Analyze Image Histograms of the CTA Datasets with the Original Size}

The image displays an object when its average intensity is different from its adjacent areas. The histogram provides an image's gray-level distribution. Multimodal histograms can occur when the image contains multiple objects of different average brightness. The CTA datasets' intensity histograms are shown in Figure. 2. There are three peaks in both of the histograms. The first one represents the background objects and its intensity is the lowest. The second one indicates the blood's intensity and its intensity is between the lowest and the highest. The third one is recognized as the objects with the highest intensities, for instance, the bones or calcified plaques, etc.

\subsection{Segment with Multiple Isovalues}

The standard marching cube algorithm is applied to extract an object's boundary point cloud. It is to find a boundary point linearly interpolated along the edge between two adjacent voxels in $x, y$, or $z$ direction. Thus the object's boundary points generated by the standard marching cube algorithm vary with the intensity threshold value defined by the user. Since the varying contrast agent among the coronary arteries, multiple threshold values, similarly as in [26], are used in this work to extract coronary artery boundaries in the marching cube algorithm. The tested CTA datasets' intensities are ranged from 0 to 4095 . These multiple threshold values are selected inside an

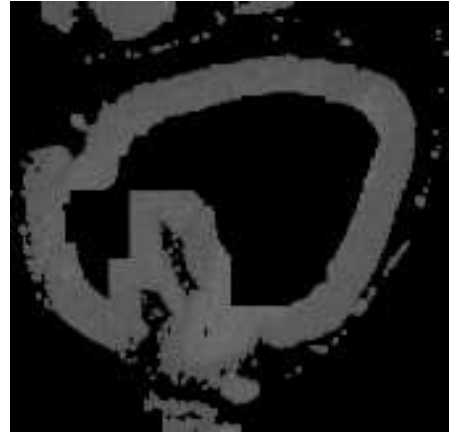

(a)

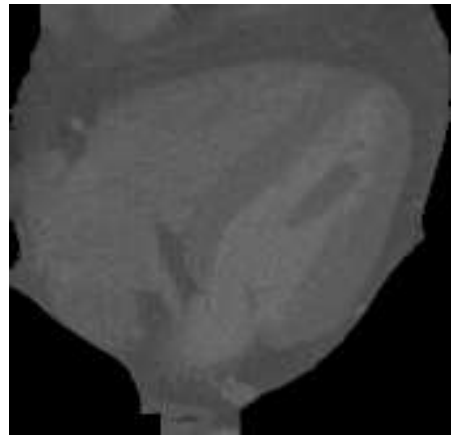

(b)

Figure. 1 (a) an image slice with the coronary artery region detected, (b) an original image slice without detection operation

intensity region around the second peak value in the CTA dataset's intensity histogram. The intensity threshold values are listed in Table I.

TABLE I THE MULTIPLE INTENSITY THRESHOLD VALUES

\begin{tabular}{|c|l|l|l|l|}
\hline Dataset & \multicolumn{4}{|c|}{ Intensity values } \\
\hline 1 & 1022 & 1102 & 1145 & 1187 \\
\hline 2 & 1343 & 1445 & 1500 & 1562.5 \\
\hline
\end{tabular}

Since multiple thresholds are used, multiple boundary layers are perhaps produced for the vessels. To reduce multiple boundary layers to one layer, the point with maximum gradient magnitude is kept among the neighboring boundary points near the gradient line of each boundary point.

\subsection{Determine the Coronary Arteries' Centerlines}

The computed boundary point cloud is tetrahedralized, centerpoints can be calculated by applying a topological analysis of the vector field based on the gradient field within every tetrahedron. The computed centerpoints can be connected via a line segment when their nearest neighboring centerpoints are found. The centerpoint can be connected with another centerpoint that is also closest to it and is in the reverse direction. Thus this centerpoint's neighbors are determined.

To differentiate the coronary artery centerpoints from the computed centerpoint cloud, one experienced medical technician can pick one seed centerpoint among the centerpoint cloud. This centerpoint is recognized as a coronary artery centerpoint; the neighboring centerpoints, which it is connected to, are recursively marked as coronary artery centerpoints. Several seed 
centerpoints with a downsampled dataset are selected to find the other portions of a coronary artery tree's centerlines. The number of seed centerpoints with an original dataset is more than a downsampled dataset.

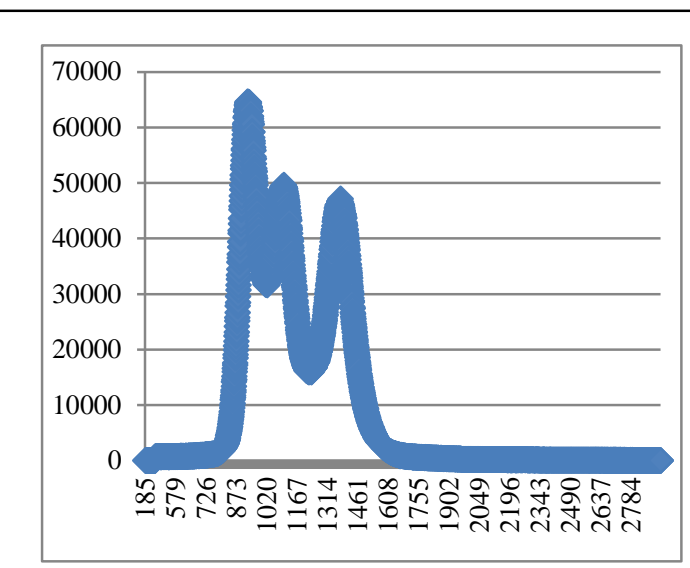

(a)

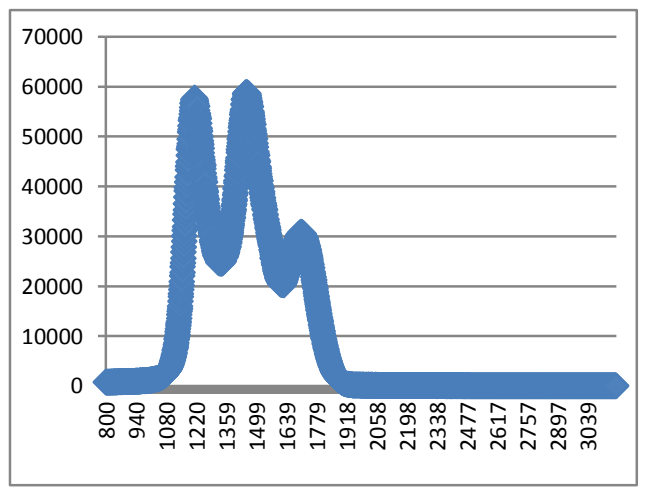

(b)

Figure. 2 intensity histograms of CTA datasets (a) dataset 1 , (b) dataset 2

\subsection{Find the Coronary Artery Boundaries}

For each coronary artery centerpoint, the boundary points are recognized as boundary points of the coronary arteries when they are enclosed in a ball with the radius of current centerpoint. These boundary points' normals must point inward of the vessel that encloses the current centerpoint. The tetrahedra attached by each of these boundary points are defined as the vessel tetrahedra except those, of which, there are any vertices' normals pointing outward of the vessel. The vertices of vessel tetrahedra are identified as the boundary points of the coronary arteries.

\subsection{Segment the CTA Dataset with the Original Size}

The coronary artery boundary voxels of the original CTA dataset can be located from those coronary artery boundary points from the downsampled CTA dataset and are dilated with a $3 \times 3 \times 3$ cube when a right artery tree is segmented and a $5 \times 5 \times 5$ cube when a left artery tree is segmented. If the voxels belong to the detected coronary artery region, they are again processed with the same procedures to the downsampled datasets, for instance, segmenting with multiple isovalues, tetrahedralizing the boundary points, determining the coronary artery centerlines and finding the coronary artery boundaries. To extract the coronary artery centerlines, the centerlines represent small vessel branches are removed manually. Thus the vessel tetrahera are defined and the coronary artery boundary points are found.
A scan-conversion algorithm [20] for lines computes the coordinates of the voxels that line on or near an ideal, infinitely thin straight line in three dimensions. In principle, the sequence of voxels is required to lie as close to the ideal line as possible and to be as straight as possible. The line segment can be drawn with a sequence of voxels described above with given start point and end point of this line segment. Scan converting polygons as area-defining primitives could be done a line segment at a time. Similarly, scan converting a tetrahedron as a volume-defining primitive can be completed a triangle at a time. Then the voxels representing a tetrahedron can be identified. And the coronary artery voxels can be obtained from all the vessel tetrahedra. The coronary artery boundary points are triangulated with a surface mesh. And the coronary artery voxels also include the voxels by the scan converting each triangle.

\section{VISUALIZE THE CORONARY ARTERY TREES}

The intensities of voxels that are not coronary artery voxels are set to zero. Those voxel sets of coronary arteries are smoothed with recursive Gaussian filter, and then are processed with the gradient based extraction algorithm. The triangle mesh is applied to the resulted boundary points to recover the surfaces of the segmented coronary artery trees.

\subsection{Rendering the Surfaces of the Coronary Artery Trees}

Two main visualization techniques for three-dimensional medical images are surface rendering and direct volume rendering (DVR) [13]. Rendering an object' surface with triangle mesh belongs to the surface rendering visualization category. In DVR, a projected two-dimensional image represents the entire three-dimensional dataset. The projected two-dimensional image does not allow DVR to capture all the three-dimensional surface information completely. Figure. 3 (a), (b), (c), (d) display the surfaces recovered by the triangle mesh on the segmented coronary artery trees. The number of boundary points, the triangles and hole triangles are listed in the Table II, 1 represents dataset 1, 2 indicates dataset 2. A hole triangle means at least one edge of the triangle connects only one triangle.

\section{TABLE II. THE TRIANGLE MESH WITH THE ARTERY TREES'} BOUNDARY POINTS

\begin{tabular}{|l|l|l|c|}
\hline $\begin{array}{l}\text { Artery } \\
\text { tree }\end{array}$ & $\begin{array}{l}\text { point } \\
\text { number }\end{array}$ & $\begin{array}{l}\text { triangle } \\
\text { number }\end{array}$ & $\begin{array}{c}\text { Hole } \\
\text { triangle } \\
\text { number }\end{array}$ \\
\hline Left 1 & 32481 & 64961 & 14 \\
\hline Right 1 & 33694 & 67400 & 0 \\
\hline Left 2 & 44189 & 88376 & 12 \\
\hline Right 2 & 27008 & 54008 & 9 \\
\hline
\end{tabular}

\subsection{Identify Calcified Plaques on the Triangle Meshed Surfaces of the Coronary Artery Trees}

For the varying contrast agent among the coronary arteries, intensities of the boundary points are assumed as with Gaussian distribution. The mean $\left(\mu_{\text {boundary point }}\right)$ and standard deviation 


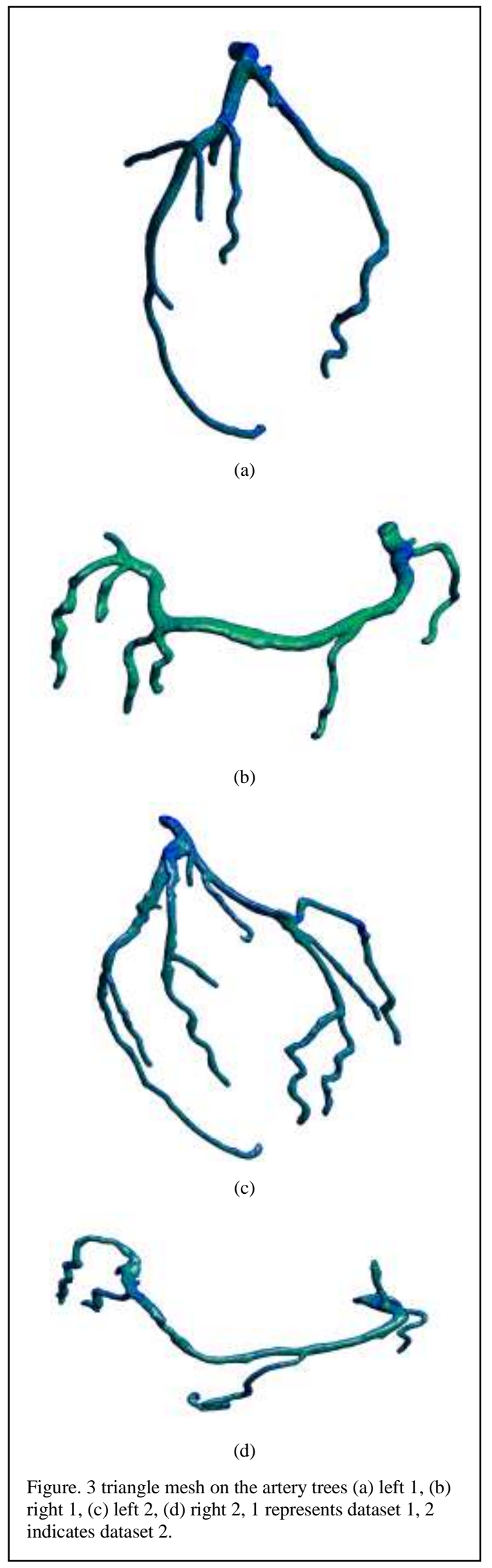

( $\left.\sigma_{\text {boundary point }}\right)$ of intensities of the boundary points are computed. Figure. 4 shows the intensities of the artery trees' boundary points mapped on the triangle mesh. The intensity values are displayed varying from low to high with color ramping map. The calcified plaques are having higher intensities compared with their neighboring intensities and are included in the vessel lumens by the multiple isovalue based algorithm. The color red represents those boundary points with intensities higher than $\mu_{\text {boundary point }}+4 \sigma_{\text {boundary point }}$. The calcified plaques can be perceived as a small block with the color red or light red if that light red's surrounding areas are colored green. Thus the calcified plaques with intensities lower than the threshold values can be identified. These calcified plaques are identified correctly as those are recognized from each image slice when the intensities of the boundary points are mapped on the triangle mesh.

Figure. 5 shows instances of the calcified plaques recognized from image slices circled with orange color. Figure. 5 (c) also displays one calcified area circled with blue color, which is not recognized with the boundary point intensity mapping, a false negative error. With tested coronary arteries, this calcified plaque detection method has zero false positive and only one false negative.

Figure. 6 visualizes the calcified plaques as sets of voxels colored orange, and they are recognized since they have higher average intensity than that of the blood of the coronary arteries. The calcified plaques are inside the vessel lumens by the multiple isovalue based algorithm and they are included in the segmented coronary artery voxels. Assume the blood intensities of the coronary arteries are with Gaussian distribution. The calcified plaques are recognized as those voxels which intensity values are greater than $\mu_{\text {voxel }}+3 \sigma_{\text {voxel }}, \mu_{\text {voxel }}$ is the mean intensity of the segmented coronary artery voxels, $\sigma_{\text {voxel }}$ is the standard deviation. The calcified plaques with intensities less than the threshold value cannot be determined. In dataset 1 and 2, there

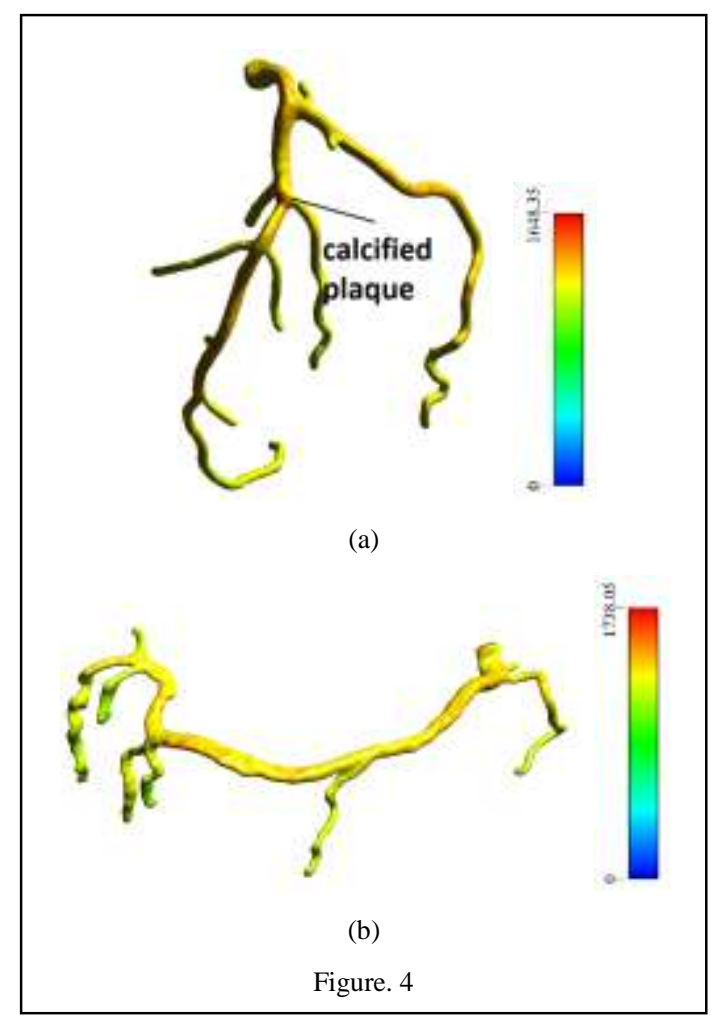



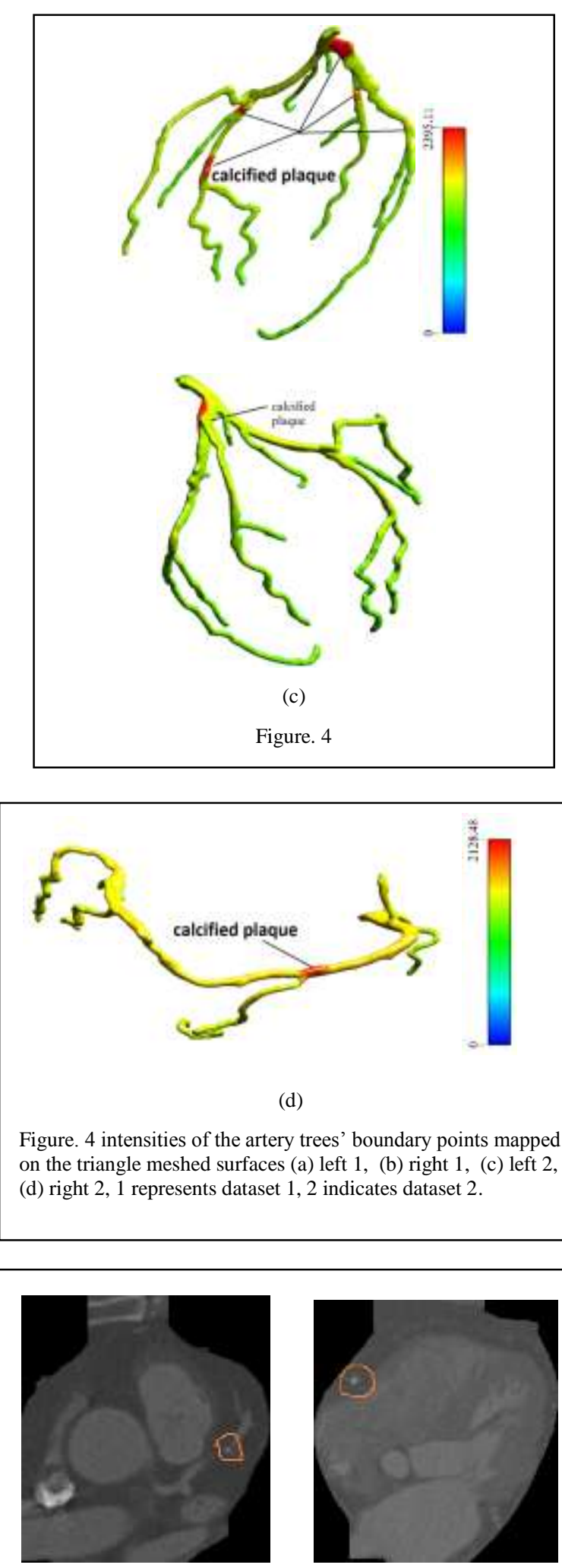

(a)

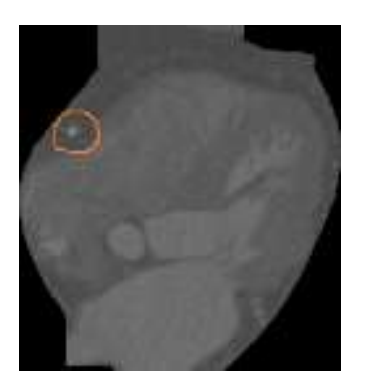

(b) (d)

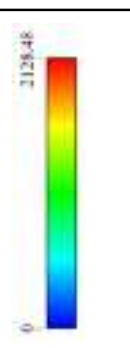
(c) left 2 , 2. 
is one false positive calcified area in the left coronary artery tree of dataset 1 , and there are still three false negative areas in the left coronary artery tree of dataset 2 .
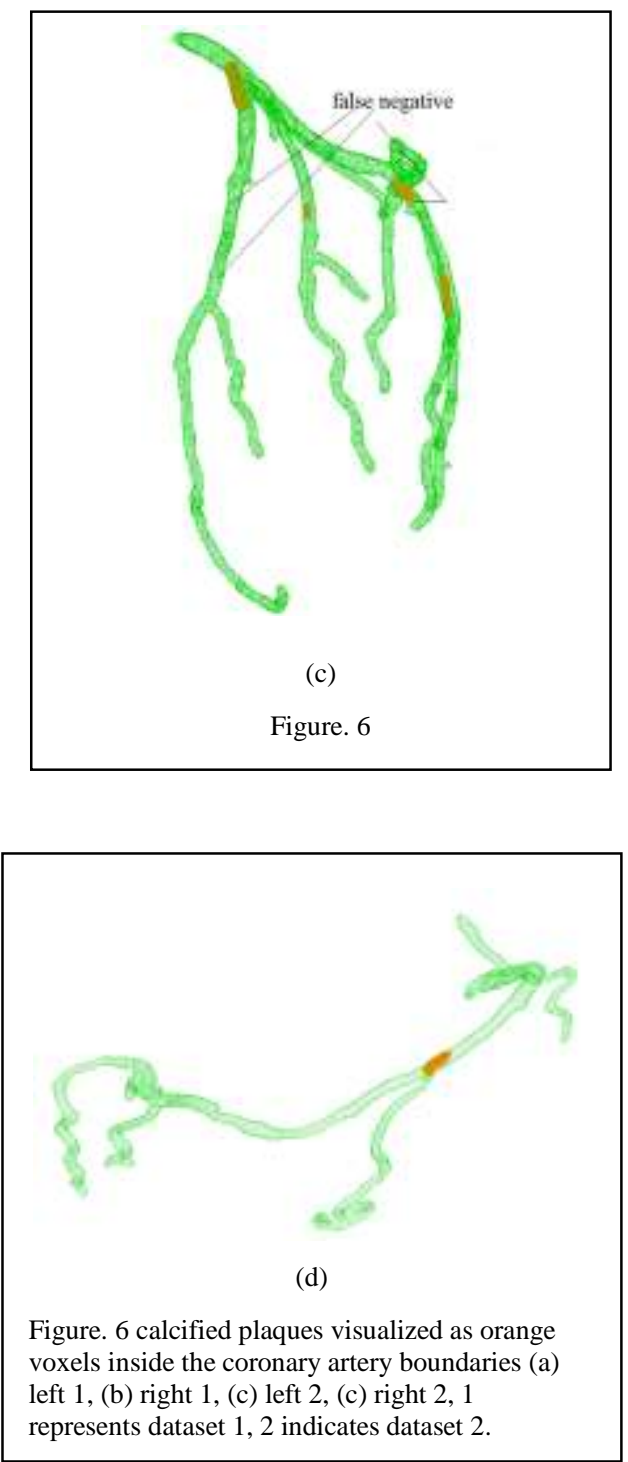

\subsection{Mapping Maximum Principle Curvatures of Boundary Points on the Triangle Meshed Surfaces of the Coronary Artery Trees}

The maximum principal curvature of a torus is a constant which is equal to the reciprocal of the minor radius [29], and the maximum principal curvature of a circular cylinder is also a constant with a value of the reciprocal of the radius of circle base [29]. This work uses the hypotheses that if the real lumen cross section structure is deviated from the circular cross section structure, different values of the maximum principle curvatures of boundary points are surrounding the lumen cross section on the triangle meshed surfaces of coronary artery trees.

According to the curvature computation in [28], the maximum principal curvatures can be derived from the local Hessian matrix at each surface triangle's vertex. They are mapped on the recovered triangle meshed surfaces of the coronary artery trees. The mean value of those maximum principle curvatures of boundary points is computed. In Figure. 7 (a), (b), (e), (f), (i), (j), $(\mathrm{m}),(\mathrm{n}),(\mathrm{q}),(\mathrm{r})$, the color blue represents those values of maximum principle curvature that are greater than the mean value. Figure. 7 (b), (f), (j), (n) show the large views of the coronary artery segments located with line segments, different colors are displayed surrounding the lumen segments. The boundary points of these artery segments show light blue or green colors for low maximum principle curvature values and blue colors for high maximum principle curvature values. The positions of high (low) maximum principle curvatures are displayed at symmetric locations around the lumen segment's axis. These artery segments are investigated by the maximum intensity projection (MIP) technique. It is one of recommended image post processing formats for interpreting the coronary CTA dataset [34]. In this work, the MIP images are parallel projected. From the three-dimensional positions where the line segments are placed, the slab volumes containing the positioned artery segments can be found. Figure. 7 (c), (d), (g), (h), (k), (l), (o), (p) are the MIP images of the slab volumes containing the vessel segments that are located with line segments. Figure. 7 (c), (g), (k), (o) are the MIP images of one side view to project the slab volume onto the projection plane where the side of colors with high values of the maximum principle curvatures can be perceived. The MIP images of another view are shown in Figure. 7 (d), (h), (l), (p), they are created when the slab volume is projected onto the projection plane where the side of colors with low values of the maximum principle curvatures can be observed. The MIP images of these two side views illustrate different radii can be obtained from these two sides views at the same cross sections of the artery segments. The different radii displayed by these MIP images agree with the different colors mapped by maximum principle curvatures. (q) is another view of mapping maximum principle curvatures on the triangle meshed surface of left coronary artery of dataset 1 . The artery segment positioned by the line segment is surrounding with blue color completely displayed in (r). The MIP images of the two side views of this artery segment in ( $\mathrm{s}$ ) and $(\mathrm{t})$ display not obvious difference of radiuses at the same cross sections of the artery segment positioned by the line segment, which agree with the same color mapped by the maximum principle curvatures.

In this work, the computed maximum principle curvatures are inspected at positions without detected calcified plaques. The tested coronary artery segments located by the line segments show that if different values of the computed maximum principle curvatures of boundary points are surrounding the same lumen cross section on the triangle meshed surfaces of coronary artery trees, the lumen displays different radiuses surrounding the same cross section by MIP images, which further indicates this cross section structure is not a circular cross section structure.

\section{CONCLUSIONS}

The method presented to segment coronary artery trees is capable to obtain the sets of voxels representing coronary arteries. The positions of the calcified plaques can be identified when the intensities of boundary points of the coronary artery trees are mapped on the triangle meshed surfaces. If different values of the computed maximum principle curvatures of boundary points surrounding the lumen cross section are mapped on the triangle meshed surfaces of the segmented coronary artery trees, the cross section structure of the coronary artery lumen segment is noncircular cross section structure. 

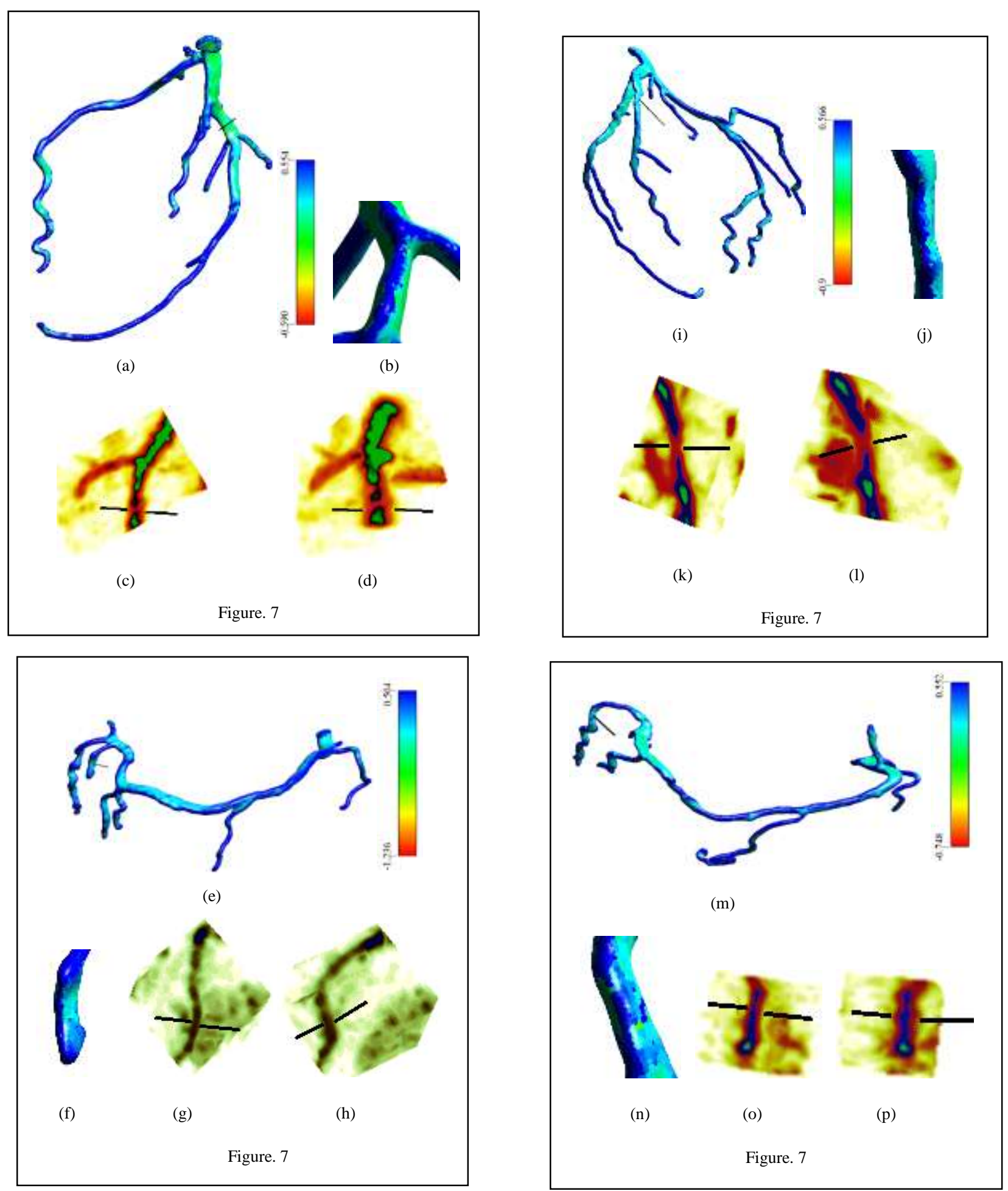


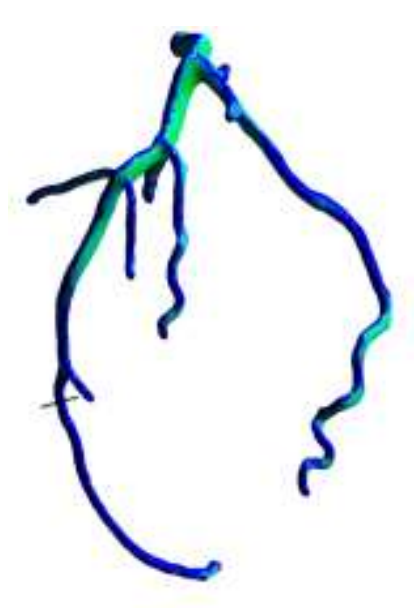

(q)

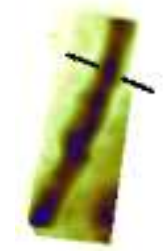

(s)

(t)

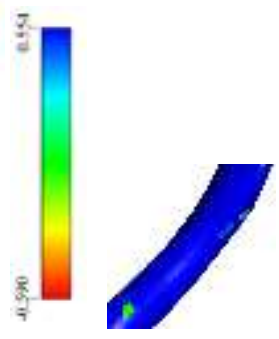

(r)

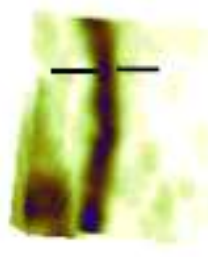

Figure. 7 maximum principle curvatures mapped on the triangle meshed surface of (a) left coronary artery of dataset 1 , (e) right coronary artery of dataset 1 , (i) left coronary artery of dataset 2, (m) right coronary artery of dataset 2; (b), (f), (j), (n) large view of the artery segment located with line segment in (a), (e), (i), (m), respectively; (c), (g), (k), (o) small radius from the MIP image in one side view of a slab volume containing the artery segment in (b), (f), (j), (n), respectively; (d), (h), (l), (p) large radius from MIP image in another side view to the same slab volume in (c), (g), (k), (o) respectively.

another view of maximum principle curvatures mapped on the triangle meshed surface of (q) left coronary artery of dataset 1 , (r) large view of the artery segment located with line segment in (q), (s) and (t) are MIP images in two sides views of the slab volume containing the artery segment located with the line segment in (q).

\section{REFERENCES}

[1] Geoffrey D. Rubin, Jonathon Leipsic, U. Joseph Schoef, Dominik Fleischmann, Sandy Napel, CT Angiography after 20 Years: A Transformation in Cardiovascular Disease Characterization Continues to Advance, Radiology, Vol 271: 3-June 2014.

[2] Paul S. Calhoun, Brian S. Kuszyk, David G. Heath, Jennifer C. Carley, Elliot K. Fishman, Spiral CT Data: Theory and Method, RadioGraphics, 19, 1999, pp745-764.

[3] Katja Bühler, Petr Felkel, Alexandra La Cruz, Geometric methods for vessel visualization and quantification - a survey, Geometric modelling for scientific visualization, G. Brunnet, B. Hamann and H. Müller (eds.), Kluwer Academic Publishers, 2004, pp. 399-420.

[4] Paul Schoenhagen, Arthur Stillman, Sandra S. Halliburton, Richard D White, CT of the Heart: Principles, Advances, Clinical Uses, Cleveland Clinic Journal of Medicine, 2, 2005.

[5] Jorge Larrey-Ruiz, Juan Morales-Sanchez, Maria C Bastida-Jumilla, Rosa M Menchon-Lara, Rafael Verdu-Monedero and Jose L
Sancho-Gomez, Automatic Image-based Segmentation of the Heart from CT Scans, EURASIP Journal on Image and Video Processing, 52, 2014.

[6] Ali Hassan, Sarfraz Ahmed Nazir, Hatem Alkadhi, Technical Challenges of Coronary CT Angiography: Today and Tomorrow, European Journal of Radiology, Vol 79, 2011, 161-171.

[7] Victoria Yeh, Rine Nakanishi, and Matthew J. Budoff, Coronary Artery Disease Progression: Insights from Cardiac CT, Current Cardiovascular Imaging Reports, 8(7), 2015.

[8] Alan Conrad bovik, Handbook of Image and Video Processing, Elesvier Science, 2005.

[9] Gregory T. Wilson, Prabhakaran Gopalakrishnan, Tahir Tak, noninvasive Cardiac Imaging with Computed Tomography, Clinical medicine \& Research, Vol 5, No. 3, 2007: 165-171

[10] T. Deschamps, P. Schwartz, D. Trebotich, P. Colella, D. Saloner, R. Malladi, Vessel segmentation and blood flow simulation using level-sets and embedded boundary methods, International Congress Series, 2004, 1268, pp. 75-80.

[11] Y. Sato, S. Nakajima, N. Shiraga, H. Atsumi, S. Yoshida, T. Koller, G. Gerig, and R. Kikinis, Three dimensional multi-scale line filter for segmentation and visualization of curvilinear structures in medical images, Medical Image Analysis, 2, 1998, pp. 143-168.

[12] A.F. Frangi, W.J. Niessen, K.L. Vincken, M.A. Viergever, Multiscale Vessel Enhancement Filtering, MICCAI'98, Lecture Notes in Computer Science, Vol 1496, pp130-137.

[13] Jayaram K. Udupa, Hsiu-Mei Hung, and Keh-Shih Chuang, Surface and Volume Rendering in Three-Dimensional Imaging: A Comparison, Journal of Digital Imaging, Vol 4, No 3 (August) 1991: pp 159-168

[14] Shawn Lankton, Arthur Stillman, Paolo Raggi and Allen Tannenbaum, Soft plaque detection and automatic vessel detection, Proceedings of Medical Image Computing and Computer Assisted Intervention (MICCAI) Workshop: Probabilistic Models for Medical Image Analysis, 2009, pp. 25-33.

[15] Felix Renard, Yongyi Yang, Coronary artery extraction and analysis for detection of soft plaques in MDTC images, IEEE International Conference on Imaging Processing (ICIP) 2008, pp 2248

[16] A. Hennemuth, T. Boskamp, D. Fritz, One-Click Coronary Tree Segmentation in CT Angiographic Images, International Congress Series, Vol. 1281, 2005, pp. 317-321.

[17] Ola Friman, Milo Hindennach, Caroline Kuhnel, Heinz-Otto Peitgen, Multiple Hypothesis Template Tracking of Small 3D Vessel Structures, Medical Image Analysis, 14 (2010) 160-171.

[18] Michel Schaap, Lisan Neefjes, Coert Metz, Alina van der Giessen, Annick Weustink, Nico Mollet, Jolanda Wentzel, Theo van Walum and Wiro Niessen, Coronary Lumen Segmentation Using Graph Cuts and Robust Kernel Regression, The Series Lecture Notes in Computer Science: Information Processing in Medical Imaging, Vol. 5636, pp 528-539.

[19] Rahil Shahzad, Hortense Kirisli, Coert Metz, Hui Tang, Michiel Schaap, Lucas van Vliet, Wiro Niessen, Theo van Walsum, Automatic Segmentation, Detection and Quantification of Coronary Artery Stenoses on CTA, International Journal Cardiovascular Imaging, 29, 2013, pp 1847-1859.

[20] James Foley, Andries Van Dam, Steven Feiner, John Hughes, Computer Graphic: Principles and Practices, $2^{\text {nd }}$ Edition, Addision-Wesley Publishing Company, Inc, 1990.

[21] William E. Lorensen, Harvey E. Cline: Marching Cubes: A high resolution 3D surface construction algorithm, Computer Graphics, 21(4), 1987, pp. 163-169.

[22] Nicole E. Jensky, Michael H. Criqui, Michael C. Wright, Christina L. Wassel, Steven A. Brody, Matthew A. Allison, Blood Pressure and Vascular Calcification, Hypertension, 2010, 55(4), 990-997.

[23] Michelle L. Frost, Rodolpho Grella, Sandrine C. Millasseau, Benyu Jiang, Geeta Hampson, Ignac Fogelman, Phil J. Chowienczyk, Relationship of Calcification of Atherosclerotic Plaque and Arterial Stiffness to Bone Mineral Density and Osteoprotegerin in Postmenopausal Women Referred for Osteoporosis Screening, Calcified Tissue International, 2008, 83, 112-120.

[24] Joshua D. Hutcheson, Natalis Maldonado, and Elena Aikawa, Small Entities with Large Impact: Microcalcifications and Atherosclerotic Plaque Vulnerability, Current Opinion in Lipidology, Vol. 25, October, 2014.

[25] Glaber S, Oeltze S, Hennemuth A, Kubisch C, Mahnken A, 
International Journal of Computer Applications Technology and Research

Volume 5-Issue 9, 603-611, 2016, ISSN:-2319-8656

Wilhelmsen S, et al. Automatic Transfer Function Specification for Visual Emphasis of Coronary Artery Plaque, Computer Graphics Forum. 2010; 29(1): 191-201

[26] Tobias Boskamp, Daniel Rinck, Florian Link, Bernd Kummerlen, Mildeberger, New Vessel Analysis Tool for Morphometric Quantification and Visualization of Vessels in CT and MR Imaging Data Sets, Radio Graphics, 24, 2004, 287-297.

[27] Rahil Shahzad, Hortense Kirisli, Coert Metz, Hui Tang, Michiel Schaap, Lucas van Vliet, Wiro Niessen, Theo van Walsum, Automatic Segmentation, Detection and Quantification of Coronary Artery Stenoses on CTA, International Journal Cardiovascular Imaging, 29 (2013), pp 1847-1859.

[28] Gordon Kindlmann, Ross Whitaker, Tolga Tasdizen and Torsten Moller, Curvature-based transfer functions for direct volume rendering: methods and applications, Proceedings of the 14th IEEE Visualization 2003, pp. 513-520.

[29] Barrett O Neil, Elementary Differential Geometry, Revised $2^{\text {nd }}$ Edition, Elsevier Academic Press Publications, 2006.

[30] Henk A. Marquering, Jouke Dijkstra Patrick J.H. de Koning, Berend C. Stoel \& Johan H.C. Reiber, Towards quantitative analysis of coronary CTA, The International Journal of Cardiovascular Imaging, 2005, 21, pp.73-84.

[31] M.J. van Gils, D. Vukadinovic, A.C. van Dijk, D.W.J. Dippel, W.J. Niessen, A. van der Lugt, Carotid Atherosclerotic Plaque Progression and Change in Plaque Composition Over Time: A 5Year Follow-Up Study Using Serial CT Angiography, American Journal of Neuroradiology, Vol 33, August 2012.

[32] P.J. de Feyter, P.W. Serruys, K. Nieman, N. Mollet, F. Cademartiri, R.J. van Geuns, C. Slager, A.F.W. van der Steen, R. Krams, J.A. Schaar, P. Wielopolski, P.M.T. Pattynama, A. Arampatzis, A. van der Lugt, E. Regar, J. Ligthart, P.Smits, Imaging of Coronary Atherosclerosis and Identification of the Vulnerable Plaque, Netherlands Hearts Journal, Vol 11, No. 9, Septmber 2003.

[33] Simone Balocco, Carlo Gatta, Marina Alberti, Xavier Carrillo, Juan Rigla, Petia Radeva, Relation between Plaque Type, Plaque Thickness, Blood Shear Stress, and Plaque Stress in Coronary Arteries Assessed by X-ray Angiography and Intravascular Ultrasound, Medical Physics, 39(12), December 2012.

[34] Jonathon Leipsic, Suhny Abbara, Stephan Achenbach, Ricardo Cury, James P. Earls, GB John Mancini, Koen Nieman, Gianluca Pontone, Gilbert L. Raff, SCCT guidelines for the interpretation and reporting of the coronary $\mathrm{CT}$ angiography: A report of the Society of Cardiovascular Computed Tomography Guildelines Committee, Journal of Cardiovascular Computed Tomography 8 , 342-358 (2014) 